\title{
Escape model for Galactic cosmic rays
}

\author{
G. Giacinti ${ }^{1,2}$, M. Kachelrie ${ }^{3}$, D. V. Semikoz ${ }^{4}$ \\ ${ }^{1}$ Max-Planck-Institut für Kernphysik, Heidelberg, Germany \\ ${ }^{2}$ University of Oxford, Clarendon Laboratory, Oxford, United Kingdom \\ ${ }^{3}$ Institutt for fysikk, NTNU, Trondheim, Norway \\ ${ }^{4}$ AstroParticle and Cosmology (APC), Paris, France
}

\begin{abstract}
We show that the cosmic ray (CR) knee can be entirely explained by energy-dependent CR leakage from the Milky Way, with an excellent fit to all existing data. We test this hypothesis calculating the trajectories of individual CRs in the Galactic magnetic field. We find that the CR escape time $\tau_{\mathrm{esc}}(E)$ exhibits a knee-like structure around $E / Z=$ few $\times 10^{15} \mathrm{eV}$ for small coherence lengths and strengths of the turbulent magnetic field. The resulting intensities for different groups of nuclei are consistent with the ones determined by KASCADE and KASCADE-Grande, using simple power-laws as injection spectra. The transition from Galactic to extragalactic CRs is terminated at $\approx 2 \times 10^{18} \mathrm{eV}$, while extragalactic CRs contribute significantly to the subdominant proton flux already for $\gtrsim 2 \times 10^{16} \mathrm{eV}$. The natural source of extragalactic CRs in the intermediate energy region up to the ankle are in this model normal and starburst galaxies. The escape model provides a good fit to $\ln (A)$ data; it predicts that the phase of the CR dipole varies strongly in the energy range between $1 \times 10^{17}$ and $3 \times 10^{18} \mathrm{eV}$, while our estimate for the dipole magnitude is consistent with observations. above $E>10^{14} \mathrm{eV}$. Adding a single nearby source to this model explains moreover the dipole anisotropy below $10^{14} \mathrm{eV}$, the specific slope of the locally measured proton flux as well as of the antiproton and positron fluxes.
\end{abstract}

The 34th International Cosmic Ray Conference,

30 July- 6 August, 2015

The Hague, The Netherlands 


\section{Introduction}

The all-particle cosmic ray (CR) energy spectrum is a nearly featureless power-law between $\sim 10^{10} \mathrm{eV}$ and $\sim 10^{20} \mathrm{eV}$, with only a few breaks in its spectral index. The two most prominent ones are the knee at $E_{\mathrm{k}} \approx 4 \mathrm{PeV}$, and the ankle at $E_{\mathrm{a}} \approx 4 \mathrm{EeV}$. For the knee, two main explanations remain possible. First, it may be the signature of the maximum energy to which Galactic CR sources can accelerate protons [1]. Second, the knee could be caused by a change in the energy dependence of the CR diffusion coefficient and thence confinement time in the Galaxy [2, 3, 4], if the CR Larmor radius is the order of the coherence length $l_{\mathrm{c}}$ of the turbulent Galactic magnetic field (GMF) at $E_{\mathrm{k}}$. In Refs. [3, 4], we studied this possibility—which we called the "escape model"-by propagating individual CRs in recent GMF models. We review here the escape model and show that it is able to explain the individual fluxes of CR groups in the entire energy region between $300 \mathrm{GeV} / \mathrm{Z}$ and the ankle. The model also satisfies the observational constraints from anisotropy data at $E>10^{14} \mathrm{eV}$. Adding a single nearby source to this model explains moreover the dipole anisotropy below $10^{14} \mathrm{eV}$ [5], the specific slope of the locally measured proton flux as well as of the antiproton and positron fluxes [6].

\section{Galactic magnetic field models and CR confinement in the Galaxy}

An important constraint on CR propagation models comes from ratios of stable primaries and secondaries produced by CR interactions on gas in the Galactic disk. In particular, the $\mathrm{B} / \mathrm{C}$ ratio has been recently measured by the AMS- 02 experiment up to $670 \mathrm{GeV} /$ nucleon [7]. In order to take advantage of the high-quality and the large energy range of the B/C data from AMS-02, we used these data to derive the grammage traversed by CRs as a function of their energies in two simple models [4]. In Fig. 1, the two sets of grammages deduced are shown with magenta and blue error-bars. Note that the error-bars take into account only the statistical and systematic errors of the AMS-02 measurement, while uncertainties in the cross sections or deficiencies of our approximations are not accounted for. The latter can be estimated by the differences between the results from the two approximations.

In order to compare these measured values to those predicted in the escape model, we inject $N$ cosmic rays at $z=0$ in the Galaxy and follow their trajectories $x_{i}(t)$ until they reach the edge of the Galaxy. Then, we calculate the average grammage $\langle X\rangle=N^{-1} c \sum_{i=1}^{N} \int d t \rho\left(x_{i}(t)\right)$ summing up the density along the trajectories of individual CRs. Since the grammage $X(E) \propto E^{-\delta}$ scales as the confinement time $\tau(E) \propto E^{-\delta}$, this quantity also serves as an indicator for changes in the CR intensity induced by a variation of the CR leakage rate.

In Fig. 1, we compare the grammage calculated from simulated CR trajectories with the grammage deduced from B/C measurements. We use the JF model for the Galactic magnetic field [8], choosing as the maximal length of the fluctuations $L_{\max }=5 l_{\mathrm{c}}=10 \mathrm{pc}$, and consider two values of its root mean square (rms) strength, the original one suggested in [8] $(\beta=1)$ and a second one rescaling it to one tenth of its original value $(\beta=1 / 10)$. (Similiar results are obtained in the GMF model of Ref. [9].) Because of the large maximum energy of the AMS-02 data, the extrapolation required from the lowest energy of our numerical calculations $\left(E=10^{14} \mathrm{eV}\right)$ to the AMS measurements has shrunk to two orders of magnitude. Using the JF model with $\beta=1$ as proposed in [8] 

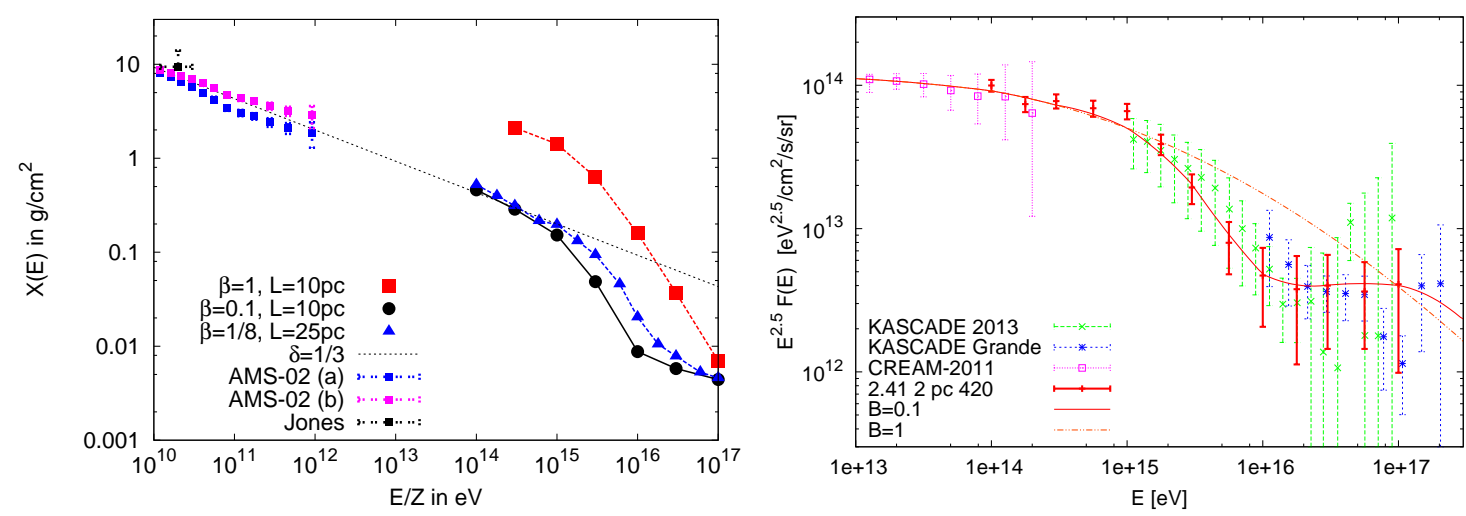

Figure 1: Left: Grammage for different coherence lengths $l_{\mathrm{c}}$ and turbulent fields: red squares $L_{\max }=10 \mathrm{pc}$ and $\beta=1$, black dots $L_{\max }=10 \mathrm{pc}$ and $\beta=0.1$, and blue triangles $L_{\max }=25 \mathrm{pc}$ and $\beta=0.125$; all cases for the JF GMF model [8]. Additionally we show the grammage deduced from B/C data. Right:Intensity using $I(E) \propto X(E)$ (red solid line) for the reduced turbulent field compared to the case of full turbulent field (red dashed line).

would require a constant power spectrum of magnetic field fluctuations, $\mathscr{P}(k) \propto k^{-\alpha}$ with $\alpha=0$, in the intermediate energy range. Such a power-spectrum is difficult to reconcile with the theoretical understanding of turbulence. Moreover, the CR spectrum is very close to a power-law above $\simeq 200 \mathrm{GV}$. This implies that if $D(E)$ would become significantly flatter beyond $\mathrm{TeV}$ energies (e.g. changing from $D(E) \propto E^{1 / 3}$ to $\left.\propto E^{0}\right)$, then the injection spectrum of sources has to have the exact opposite change of slope (e.g. respectively from $\propto E^{2.4}$ to $\propto E^{2.7}$ ). Alternatively, a change in the source density should compensate the change in $D(E)$ such that the observed CR intensity remains a nearly featureless power-law [10]. Although such a conspiracy cannot be excluded, it appears to us as a not very attractive option. We choose a Kolmogorov power-spectrum $\mathscr{P}(k) \propto k^{-5 / 3}$ as the theoretical model with the smallest slope $\alpha$, and have therefore to reduce $B_{\mathrm{rms}}$ by a scaling factor $\beta<1$. Next we examine how the shape of the grammage $X$ as function of energy $E / Z$ depends on the two parameters $l_{c}$ and $\beta$. In [3], we discovered a specific shape of $X(E)$ that leads not only to a knee-like feature but reproduced also the recovery of the proton and helium spectra above $E / Z \sim 10^{16} \mathrm{eV}$, visible in the KASCADE-Grande data. It is clear that a too strong turbulent field, $\beta \sim 1$, results in knee-like feature at too high energy. Compensating a relatively strong turbulent field by decreasing the coherence length tapers off both the knee-like feature and the recovery. As a consequence, the allowed range of turbulent field strengths and coherence lengths is correlated and very restricted, $l_{\mathrm{c}} \simeq(1-10) \mathrm{pc}$ and $\beta \simeq 1 / 10-1 / 8$. In the right panel of Fig. 1 , we show the resulting CR proton intensity (knee and recovery) for $L_{\max }=10 \mathrm{pc}$ and $\beta=1 / 10$ (red solid line).

\section{Fluxes of Galactic CR groups}

For the calculation of the CR flux at Earth, we adapt a procedure based on the use of templates as decribed in detail in Ref. [4]. Results for nuclei with charge $Z$ are deduced from the calculations for protons by shifting the energy by a factor $Z$. We then interpolate the resulting CR nuclei fluxes to the same energies as for protons. At energies below $Z \times 100 \mathrm{TeV}$, we assume that diffusion in the Kolmogorov turbulence shifts the injection power-law $\propto E^{-\alpha}$ by $1 / 3$ to the spectrum $\propto E^{-\alpha-1 / 3}$ 

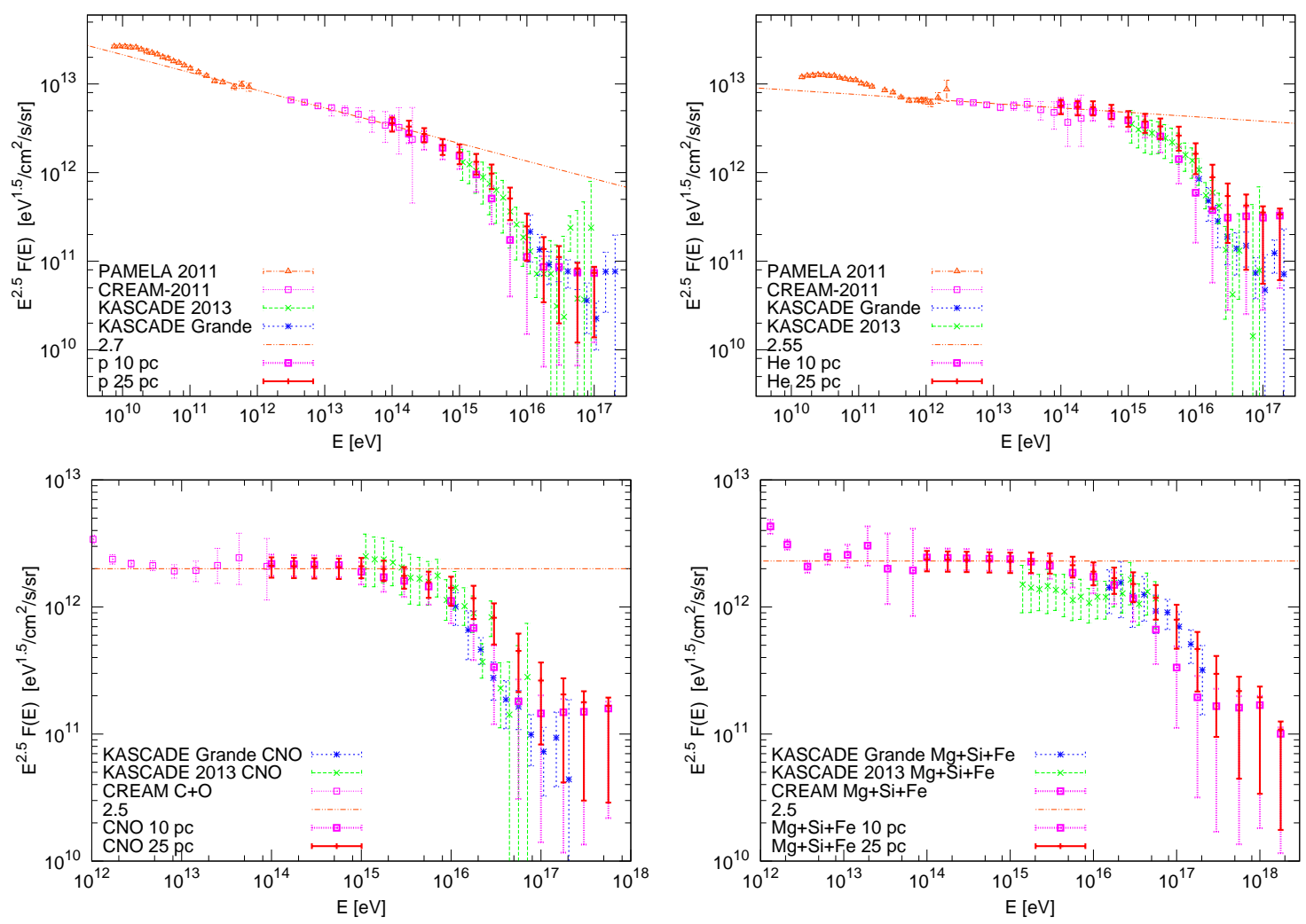

Figure 2: Fluxes of CR protons (upper left), helium (upper right), CNO (lower left) and heavy elements including $\mathrm{Mg}, \mathrm{Si}$ and $\mathrm{Fe}$ (lower right) are shown in red. Errorbars show the variations in time of the fluxes.

observed. This is indeed what we observe in our simulations in the energy range $\simeq Z \times(100-$ 300) $\mathrm{TeV}$. Therefore we assume that the CR spectrum of protons released by sources follows a power-law spectrum $\propto E^{-2.4}$; the maximal energy $E_{p}$ of protons will be fixed later by considering constraints form the resulting dipole anisotropy and the observed nuclear composition of CRs. For all other nuclei, we use power-law spectra with either $\propto E^{-2.17}$ or $\propto E^{-2.22}$ and maximal energy $Z E_{p}$. These power-law indices are chosen so as to fit the direct observations from CREAM at low energy. We fix the density of sources by normalizing the flux found in our simulations to the observed one at $100 \mathrm{TeV}$. On average, we require 130 sources per $100 \mathrm{kyr}$ for a total energy per source of $E_{\mathrm{tot}}=10^{50} \mathrm{erg}$, so as to fit the observed CR spectra.

In Fig. 2, we plot the CR nuclei fluxes, multiplied by $E^{2.5}$, as a function of energy. In the upper left and upper right panels of Fig. 2, we show the proton and helium fluxes, both for turbulent fields with $L_{\max }=10 \mathrm{pc}$ and with $L_{\max }=25 \mathrm{pc}$. We plot orange lines $\propto E^{-2.4-1 / 3}$ (upper left panel) and $\propto E^{-2.22-1 / 3}$ (upper right panel), which represent the slopes expected theoretically at Earth, for our injection spectra with $\alpha=-2.4$ and -2.22 and Kolmogorov turbulence. Note that the slopes of the injection spectra required for nuclei, $\alpha \simeq 2.2$, coincide with the naive expectations from diffusive shock acceleration. Only the proton injection spectra requires a somewhat harder slope, $\alpha=2.4$.

In the two upper panels, we show the experimental data from PAMELA [11] (orange points), CREAM [12] (magenta), KASCADE [13] (green) and KASCADE-Grande [13] (blue). The proton flux reported by KASCADE-Grande is $40 \%$ larger than the flux from KASCADE in the 
$(10-30) \mathrm{PeV}$ region, where errorbars of both experiments are relatively small. In contrast, the helium flux from KASCADE-Grande is below the one measured by KASCADE. This behavior is explained by the insufficient discrimination power between protons and helium in the KASCADEGrande experiment [14]. Therefore we chose to reduce the proton flux of KASCADE-Grande by $40 \%$, and add this difference to the helium flux, in same energy bins. By doing so, the CR fluxes of KASCADE-Grande and KASCADE experiments become consistent with each other. In the lower left panel of Fig. 2, we plot the CNO flux, which predominantly consists of carbon and oxygen. We calculate the carbon and oxygen fluxes by normalizing them to the CREAM fluxes interpolated to higher energies with power-laws, and then sum them up. The CREAM flux in this figure is the sum of its carbon and oxygen fluxes, where we use carbon energy bins for the binning, and interpolate the oxygen flux to these bins before summing up. KASCADE and KASCADE-Grande measurements of the CNO flux are directly compared to our fluxes. In the lower right panel of Fig. 2, we show the flux of heavy nuclei, which is dominated by $\mathrm{Mg}$, Si and Fe nuclei.

As can be seen in Fig. 2 (upper right), the CR proton flux follows a power-law from $300 \mathrm{GeV}$ up to about $1 \mathrm{PeV}$. It then changes to a steeper slope at the knee, and recovers at $\simeq 10 \mathrm{PeV}$ to a flatter power-law with index $\alpha \simeq 2.5$. Similar 'knee-like' cutoffs, shifted by factors $Z$ in energy, are visible in the fluxes of all groups of CR nuclei-see the other panels of Fig. 2. These plots demonstrate that the escape model fits very well all these observations. As discussed previously in [3], the knee is due, in this model, to a change in behaviour with energy of the CR diffusion coefficient. The energy of the knee corresponds to the energy at which the Larmor radius of CR protons is of the order of the coherence length of the turbulent magnetic field $\left(l_{\mathrm{c}}=L_{\max } / 5\right.$ for a Kolmogorov spectrum). For the field strengths we consider in this paper, $B_{\mathrm{rms}} \simeq 0.3 \mu \mathrm{G}$ (or $\beta=0.1 / 8$ ) close to the solar system, we find in our calculations a change in the slope of the CR flux at about $1 \mathrm{PeV}$, as observed in the proton data.

Let us note that, in this model, the flatter part of the CR proton flux above $\simeq 10 \mathrm{PeV}$ is dominated by recent nearby sources. This is due to the fact that the confinement time of CR protons in the Galaxy quickly drops with energy beyond the energy of the knee. Therefore fluctuations with time (on $\simeq(10-100)$ kyr time scales) of the CR proton flux at Earth are large above $10 \mathrm{PeV}$-what explains the large error bars of the theoretical prediction at high energies.

\section{CR dipole anisotropy and single nearby source}

In addition to fitting the above observables, we still have to verify that the model presented here is also consistent with the existing upper limits on the CR anisotropy. In the diffusion approximation, the CR dipole anisotropy $d$ is given by $d=3 D \nabla \ln (n) / c$. Following the same procedure as in [3], we compute the average anisotropy and derive the energy dependence of $D(E)$ from the escape rate as calculated previously, setting $D(E / Z) \propto 1 / \tau_{\mathrm{esc}}(E / Z)$. We fix the proportionality constant by requiring that the dipole amplitude $d=\sum_{k} f_{k} d_{k}$ equals the dipole component $\tilde{d}$ observed by the EAS-TOP collaboration at $E=1.1 \times 10^{14} \mathrm{eV}$ [18]. Here, $k$ labels the groups of nuclei we consider in the Galactic flux plus an extragalactic component. The latter has a dipole amplitude which is independent of its composition and which we set equal to $0.6 \%$, as expected for the extragalactic Compton-Getting effect [22]. The factor $f_{k}$ corresponds to the fraction the component $k$ contributes to the total CR flux, and $d_{k} \propto 1 / \tau_{\text {esc }}(E / Z)$ to their individual dipole. 

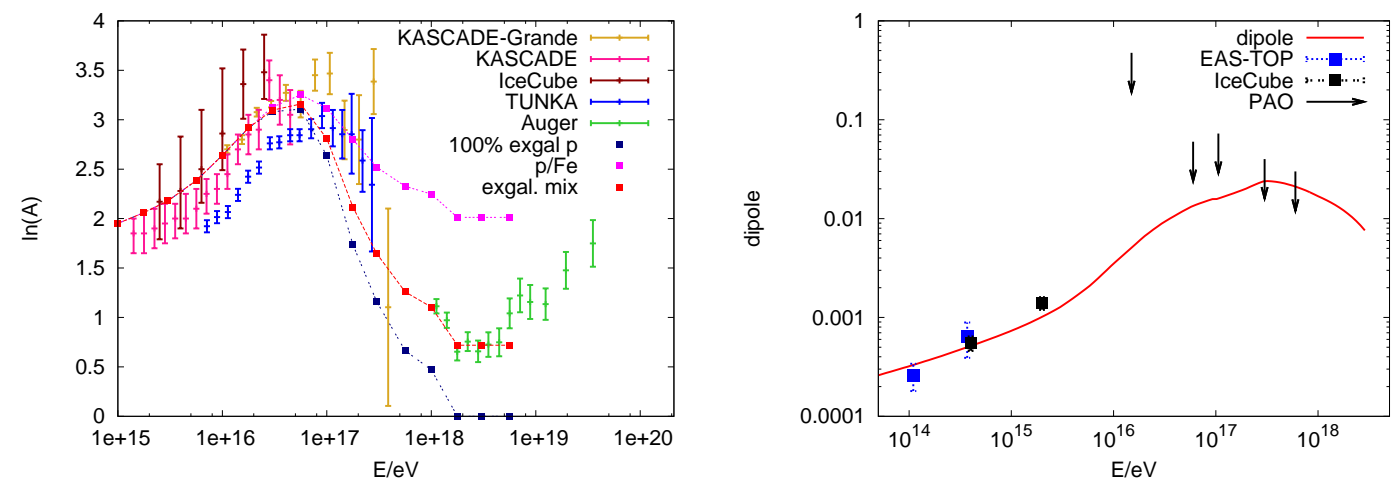

Figure 3: Left: Dipole amplitude $d(E)$ as a function of energy $E$ in the GMF model of Ref. [8], using a reduced turbulent magnetic field with $\beta=1 / 8$ and $L_{\max }=25 \mathrm{pc}$. Right: Average of the logarithmic mass In A predicted by our model for three different assumptions on the composition of extragalactic CRs, versus the experimental data.

In the right panel of Fig. 3, we show the resulting dipole amplitude $d$ as a function of energy $E$. As expected, the amplitude raises below the knee as $E^{1 / 3}$, while it increases approximately as $E^{0.7}$ until $1 \times 10^{17} \mathrm{eV}$. At higher energies, the dipole amplitude decreases, which is due to the facts that the Galactic composition becomes heavier and that the extragalactic contribution grows. We also plot the values of $\tilde{d}$ observed by IceCube [19], as well as the 99\% C.L. upper limits on $d_{\perp}$ from the Pierre Auger Observatory [20]. Comparing our estimate for the dipole amplitude with the upper limits in the energy range $10^{17}-10^{18} \mathrm{eV}$, we should take into account that the approximation $d \propto 1 / \tau_{\text {esc }}(E / Z)$ starts to break down above $E / Z \gtrsim 10^{17} \mathrm{eV}$, which leads to a sizeable error. We conclude therefore that our prediction is marginally consistent with these limits. The Pierre Auger Observatory should however be able to reach a detection of the dipole anisotropy. Let us also note that the escape model predicts that the phase of the dipole amplitude varies strongly in the energy range between $1 \times 10^{17}$ and $3 \times 10^{18} \mathrm{eV}$ : This corresponds to the range where the transition from Galactic to extragalactic CRs lies. Such a picture is supported by current observations of the phase of the dipole, see References [18, 19, 20].

In summary, there are two reasons for having an early transition, from predominantly Galactic to predominantly extragalactic CRs, at $E \approx$ a few $\times 10^{17} \mathrm{eV}$. First, the limits on the observed dipole anisotropy requires either a very heavy Galactic composition or a predominantly extragalactic contribution at $E \gtrsim 10^{18} \mathrm{eV}[23,20]$. The former possibility is however strongly disfavored by the recent composition measurements from the Auger collaboration [16, 17]. Second, identifying the energy where $\ln (A)$ stops to decrease with the maximum energy to which Galactic sources can accelerate iron, $E_{\max , \mathrm{Fe}} \approx 3 \times 10^{18} \mathrm{eV}$, suggests that the maximal rigidity reached in Galactic sources satisfies $\mathscr{R}_{\max }=E_{\text {max }, \mathrm{Fe}} /(26 e) \sim 10^{17} \mathrm{~V}$. Note that the cutoff in the Galactic CR spectrum would be at lower energies, if no nearby active CR source exists [4]. For more details on the transition from Galactic to extragalactic CRs, and on the fluxes of secondary gamma-rays and high-energy neutrinos from star-forming galaxies in the escape model, we refer the reader to [24].

Finally, we comment briefly on an extension of the escape model where the effects of a recent, local SN are added [5, 6]. In Ref. [6], it was shown that a nearby CR source which was active 
$\sim 2$ Myr ago and has injected $(2-3) \times 10^{50}$ erg in CRs explains naturally the excess of positrons and antiprotons above $\sim 20 \mathrm{GeV}$ as well as the discrepancy in the slopes of the spectra of CR protons and heavier nuclei in the $\mathrm{TeV}-\mathrm{PeV}$ energy range. The transient nature of the source and its overall energy budget point to a $\mathrm{SN}$ origin. The age of the SN suggests that the local CR injection was produced by the same $\mathrm{SN}$ that has deposited ${ }^{60} \mathrm{Fe}$ isotopes in the deep ocean crust. In Ref. [5], it was argued that the observation of a constant dipole anisotropy indicates that a single source dominates the CR flux in the corresponding energy range. A natural explanation for the observed plateau between $2-20 \mathrm{TeV}$ in the $\mathrm{CR}$ anisotropy is thus the presence of a single, nearby source. Fixing the source age to $\sim 2 \mathrm{Myr}$ as suggested by the explanation of the antiproton and positron data through a local source, the source distance follows as $\sim 200 \mathrm{pc}$. This value should be compared to the range a few hundred pc estimated from the CR flux level in Ref. [6]. Combined with the contribution of the global CR sea calculated in the escape model, this allows for the first time to explain qualitatively the data for the dipole anisotropy.

\section{Conclusions}

We have shown that the knee can be entirely explained by energy-dependent CR leakage from the Milky Way, with an excellent fit to all existing data from $E / Z \sim 300 \mathrm{GeV}$ to $100 \mathrm{PeV}$. In particular, all deviations from a single power-law behavior that are observed in the CR intensity of individual CR groups in the energy range $E / Z \sim 200 \mathrm{GeV}$ up to $100 \mathrm{PeV}$ are consistently explained by rigidity-dependent $C R$ escape. This model requires small coherence lengths of the turbulent field and relatively small turbulent magnetic field strengths. If these two conditions are fullfilled, then the CR escape time $\tau_{\text {esc }}(E)$ exhibits a knee-like structure around $E / Z=$ few $\times 10^{15} \mathrm{eV}$ together with a recovery around $E / Z \simeq 10^{16} \mathrm{eV}$. Let us note that a reduced turbulent GMF strength would facilitate the search for the sources of ultra-high energy CRs [25].

We have determined the maximal rigidity $\mathscr{R}_{\max }=E_{\max , \mathrm{Fe}} /(26 e) \sim 10^{17} \mathrm{~V}$ to which Galactic $\mathrm{CR}$ sources are able to accelerate CRs by identifying it with the energy where $\ln (A)$ derived from PAO measurements stops to decrease. The resulting flux ratio of Galactic and extragalactic sources is in our model 1:1 at $E_{*} \approx 2 \times 10^{17} \mathrm{eV}$, dropping to $0: 1$ at $2 \times 10^{18} \mathrm{eV}$. The extragalactic CR flux in the intermediate energy region up to ankle should be composed mainly of CRs accelerated in starbust galaxies. Since the transition from Galactic to extragalactic CRs happens in this model at rather low energies, the estimated CR dipole anisotropy is consistent within uncertainties with upper limits in the energy range $10^{17}-10^{18} \mathrm{eV}$, while it reproduces the measurements at lower energies from EAS-TOP and IceCube. The dipole phase is expected to change between $1 \times 10^{17}$ and $3 \times 10^{18} \mathrm{eV}$, i.e. the energy range of the transition from Galactic to extragalactic CRs. Such a behavior corresponds to the one observed, providing thus additional evidence for a transition from Galactic to extragalactic CRs in this energy region.

\section{Acknowledgments}

GG acknowledges funding from the European Research Council under the European Community's Seventh Framework Programme (FP7/2007 - 2013)/ERC grant agreement no. 247039. The work of DS was supported in part by grant RFBR \# 13-02-12175-ofi-m. 


\section{References}

[1] A. M. Hillas, J. Phys. G 31, R95 (2005) and references therein.

[2] V. L. Ginzburg and S. I. Syrovatskii, The Origin of Cosmic Rays (Pergamon Press, 1964); V. S. Ptuskin et al., Astron. Astrophys. 268, 726 (1993); J. Candia, E. Roulet and L. N. Epele, JHEP 0212, 033 (2002); J. Candia, S. Mollerach and E. Roulet, JCAP 0305, 003 (2003).

[3] G. Giacinti, M. Kachelrieß and D. V. Semikoz, Phys. Rev. D 90, R041302 (2014) [arXiv:1403.3380 [astro-ph.HE]].

[4] G. Giacinti, M. Kachelrieß and D. V. Semikoz, Phys. Rev. D 91, 083009 (2015) [arXiv:1502.01608 [astro-ph.HE]].

[5] V. Savchenko, M. Kachelrieß and D. V. Semikoz, arXiv:1505.02720 [astro-ph.HE].

[6] M. Kachelrieß, A. Neronov and D. V. Semikoz, arXiv:1504.06472 [astro-ph.HE].

[7] A. Oliva et al. [AMS-02 Collaboration], to appear in proc. of the 33rd ICRC (2013) [ID 1266].

[8] R. Jansson and G. R. Farrar, Astrophys. J. 757, 14 (2012); Astrophys. J. 761, L11 (2012).

[9] M. S. Pshirkov, P. G. Tinyakov, P. P. Kronberg and K. J. Newton-McGee, Astrophys. J. 738, 192 (2011) [arXiv:1103.0814 [astro-ph.GA]].

[10] M. Kachelrieß and D. V. Semikoz, Phys. Lett. B 634, 143 (2006) [astro-ph/0510188].

[11] O. Adriani et al. [PAMELA Collaboration], Science 332, 69 (2011).

[12] Y. S. Yoon et al., Astrophys. J. 728, 122 (2011).

[13] W. D. Apel et al. [KASCADE-Grande Collaboration], Astropart. Phys. 47, 54 (2013).

[14] A. Haungs, private communication.

[15] R. Abbasi et al. [IceCube Collaboration], Astropart. Phys. 42, 15 (2013).

[16] A. Aab et al. [Pierre Auger Collaboration], [arXiv:1409.4809 [astro-ph.HE]].

[17] A. Aab et al. [Pierre Auger Collaboration], [arXiv:1409.5083 [astro-ph.HE]].

[18] M. Aglietta et al. [EAS-TOP Collaboration], Astrophys. J. 692, L130 (2009).

[19] R. Abbasi et al. [IceCube Collaboration], Astrophys. J. 746, 33 (2012).

[20] P. Abreu et al. [Pierre Auger Collaboration], Astrophys. J. 762, L13 (2012); Astrophys. J. Suppl. 203, 34 (2012); I. Sidelnik et al. [Pierre Auger Collaboration], to appear in proc. of the 33rd ICRC (2013).

[21] M. G. Aartsen et al. [IceCube Collaboration], Astrophys. J. 765, 55 (2013).

[22] M. Kachelrieß and P. D. Serpico, Phys. Lett. B 640, 225 (2006).

[23] G. Giacinti, M. Kachelrieß, D. V. Semikoz and G. Sigl, JCAP 1207, 031 (2012).

[24] G. Giacinti, M. Kachelrieß and D. V. Semikoz, Proc. of the 34th ICRC, contribution ID 709.

[25] G. Giacinti, M. Kachelrieß, D. V. Semikoz and G. Sigl, Astropart. Phys. 35, 192 (2011). 